\title{
Intraosseous Injections Are Safe And Effective in Knee Osteoarthritis: A Systematic Review
}

\author{
Brjan Kaiji Betzler, M.B.B.S., Yan-Yu Julius Chee, M.B.B.S., and \\ Hamid Rahmatullah Bin Abd Razak, M.B.B.S., M.Med. (Ortho), F.R.C.S.Ed. (Orth)
}

\begin{abstract}
Purpose: To evaluate clinical outcomes after intraosseous injection for knee osteoarthritis systematically with available clinical evidence. Methods: A systematic search methodology of the PUBMED, EMBASE, and CINAHL databases was conducted in November 2020. The search workflow was in adherence to the Preferred Reporting Items for Systematic Reviews and Meta-Analyses (PRISMA). The following inclusion criteria were adopted: clinical trials of any level of evidence, reporting clinical outcomes following intraosseous injections of bone substitutes or biologic agents, and mesenchymal stem cells or platelet-rich plasma into the knee as treatment modalities for osteoarthritis. Duplicate data and articles not written in English were excluded from this review. Results: Six studies were identified and included in this review, with a total of 167 patients. Two studies used subchondroplasty CaP injections, while 4 studies used intraosseous injections of platelet-rich plasma. Two studies provided Level II evidence, 2 studies provided Level III evidence, and a further 2 provided Level IV evidence. Five out of 6 studies reported data using the visual analog scale, 4 studies used the Knee Injury and Osteoarthritis Outcome Score, while 3 studies used the Western Ontario and McMaster Universities Osteoarthritis Index. Clinical improvements in pain and functionality were documented in all trials, with only a few patients experiencing adverse events. Conclusion: Intraosseous injections for knee osteoarthritis are safe and effective. However, multiple pertinent variables such as safety, cost of treatment, and performance against placebos and other treatment modalities require further evaluation before intraosseous injections can be considered as standard treatment for patients presenting with osteoarthritis of the knee.
\end{abstract}

\section{Introduction}

$\mathbf{O}$ steoarthritis (OA) is a progressive and degenerative disease involving the cartilage and its surrounding tissue. ${ }^{1}$ It is one of the commonest chronic diseases worldwide and imposes a significant economic burden on patients and society. ${ }^{2}$ In particular, OA of the hips and knees tends to cause the greatest burden to the

From Lee Kong Chian School of Medicine, Nanyang Technological University, Singapore (B.K.B., J.C.Y.Y.); Department of Orthopaedic Surgery, Sengkang General Hospital, Singapore (H.R.B.A.R.); and SingHealth Duke-NUS Musculoskeletal Sciences Academic Clinical Programme, Singapore (R.B.A.R.).

Full ICMJE author disclosure forms are available for this article online, as supplementary material.

Received January 15, 2021; accepted June 28, 2021.

Address correspondence to Hamid Rahmatullah Bin Abd Razak, M.B.B.S., M.Med. (Ortho), F.R.C.S.Ed. (Orth), Department of Orthopaedic Surgery, Sengkang General Hospital 110 Sengkang East Way, Singapore 544886. E-mail:hamidrazak@gmail.com

(C) 2021 THE AUTHORS. Published by Elsevier Inc. on behalf of the Arthroscopy Association of North America. This is an open access article under the CC BY-NC-ND license (http://creativecommons.org/licenses/by-nc-nd/4.0/). 2666-061X/2150

https://doi.org/10.1016/j.asmr.2021.06.006 population, as deterioration of these large weightbearing joints severely impact the ability to carry out activities of daily living. The number of people affected by OA globally rose by $48 \%$ from 1990 to 2019 and was ranked the 15th highest cause of years lived with disability (YLDs) worldwide, accounting for $2 \%$ of the total global YLDs. ${ }^{3,4}$ Currently, there is no definitive cure for OA, and most treatments strive to alleviate pain and delay functional decline. Total knee replacement (TKR) is a quick efficacious solution for severe debilitating knee OA that is unresponsive to medical treatment, but it has poor sustainability in the long run, especially in younger patients because of the increased need for revision surgeries. Although $\mathrm{OA}$ is a disease that mainly targets older adults, it is critical to recognize that more than half of the people diagnosed with symptomatic knee OA are under 65 years of age, 5,6 which could be attributed by worldwide obesity rates nearly tripling since 1975, as estimated by the World Health Organization. ${ }^{7}$ Bayliss et al. ${ }^{8}$ described the lifetime risk of requiring revision surgery in patients who had total hip replacement or total knee replacement younger than the age of 70 years at about $35 \%$, significantly higher than patients over the age of 70 . 
Therefore, TKR is a poor treatment of choice for patients in the younger age groups.

Traditionally, OA was considered to be a disease caused by wear and tear of the articular cartilage; however, recent evidence suggests that subchondral bone and synovial inflammation can lead to the initiation and progression of the disease. ${ }^{9}$ Studies into OA pathogenesis demonstrated that extensive injury to the cartilage causes structural instability, and the release of inflammatory cytokines, such as interleukin (IL)-1 $\beta$ and tumor necrosis factor (TNF)-a, which disrupt the tissue homeostasis pathways of the osteochondral unit and triggers matrix degradation. ${ }^{21}$ Intra-articular (IA) injections address some of these complications, but studies have shown that some patients, particularly those with advanced knee OA, such as Ahlback Type III and above, do not respond to treatment with IA infiltrations of PRP, with the treatment results worsening with increasing OA severity. ${ }^{10-12}$ The absence of response could be because the subchondral bone is not a target of IA drug delivery. Considering the involvement of subchondral pathology in OA and the limitations of IA injections, intraosseous (IO) treatment modalities such as IO injections of platelet-rich plasma (PRP) and subchondroplasty (SCP) are promising, as they are thought to provide a supportive environment for cartilage repair by targeting proinflammatory mediators and improving structural integrity in the subchondral bone. ${ }^{13}$ However, the role of subchondral pathology in OA is still poorly understood, and the efficacy of biologics in treating the disease is still widely debated. The purpose of this study is to evaluate clinical outcomes after intraosseous injection for knee osteoarthritis systematically with available clinical evidence. We hypothesize that intraosseous injections will be safe and effective for knee osteoarthritis.

\section{Methods}

\section{Eligibility Criteria}

The inclusion criteria adopted for study selection were as follows: clinical trials of any level of evidence, reporting clinical outcomes following IO injections of bone substitutes or biologic agents, and mesenchymal stem cells (MSCs) or platelet-rich plasma (PRP) into the knee as treatment modalities for osteoarthritis, with a minimum number of 10 patients treated. Case reports, review articles, published abstracts, studies involving less than 10 patients, and duplicate data (the most recent series was included) were excluded from this review. Articles not written in English, or where access to the full text was unavailable, were also excluded.

\section{Information Sources and Study Selection}

An electronic search was performed by 2 independent authors (B.K.B. and J.C.Y.Y.) in the PUBMED,
EMBASE, and CINAHL databases. All relevant studies in each database, published up to November 8, 2020, were identified. The following search string was used to query citation titles and abstracts: "(Subchondroplasty OR SCP OR [intra-osseous] OR [intraosseous]) AND ([injection] OR [injections]) AND ([osteoarthritis] OR [osteo-arthritis]) AND knee OR ([Subchondroplasty OR SCP] AND ([osteoarthritis] OR [osteo-arthritis]) AND knee)". This review was not registered on the PROSPERO database. The search workflow was in adherence to the Preferred Reporting Items for Systematic Reviews and Meta-Analyses (PRISMA), ${ }^{14}$ and is shown in Fig 1.

The articles were assessed independently by 2 authors (B.K.B. and J.C.Y.Y.) to determine eligibility for inclusion in the analysis. Any disagreements were resolved by consensus discussion among the authors. A total of 6 studies were included in the final review.

\section{Data Collection}

All data from the texts, figures, and tables of the included studies were extracted to Microsoft Excel spreadsheet software for analysis and review. The specific information extracted included the following: 1) study details, including study design and level of evidence; 2 ) study population details, including number of patients, the size of the control group (if any), and the surgical procedures performed; 3) objective of study; 4) intervention instituted; 5) system used and composition and quality of PRP (if PRP Used); 6) outcomes studied and criteria/scores used to quantify them; and 7) results and any reported complications.

\section{Quality Assessment of Studies}

The quality of the single RCT included in this study was assessed using the Cochrane Collaboration risk assessment tool. ${ }^{15}$ The Risk of Bias in Non-Randomised Studies-of Intervention (ROBINS-I) tool ${ }^{16}$ was also used in the evaluation of the quality of evidence for each outcome measure. The results of the Quality Assessment are detailed in Table 1.

\section{Results}

The six studies ${ }^{17-23}$ included a total of 167 patients, with the most common follow-up time points being 6 months and 12 months postinjection. Two studies used subchondroplasty CaP injections (SCP; Zimmer Knee Creations, Warsaw, IN, USA) as a treatment modality, while 4 studies used IO Injections of PRP.

A total of 104 records were identified, of which 54 remained after removal of duplicates. Following title and abstract screening, 13 articles were identified and assessed in full text screening. Six articles were then excluded because of a wrong intervention, or the full texts were unavailable. Two articles were determined to be follow-ups addressing the same study, and, thus, were merged into a single study for our consideration. 
Fig 1. PRISMA flowchart.

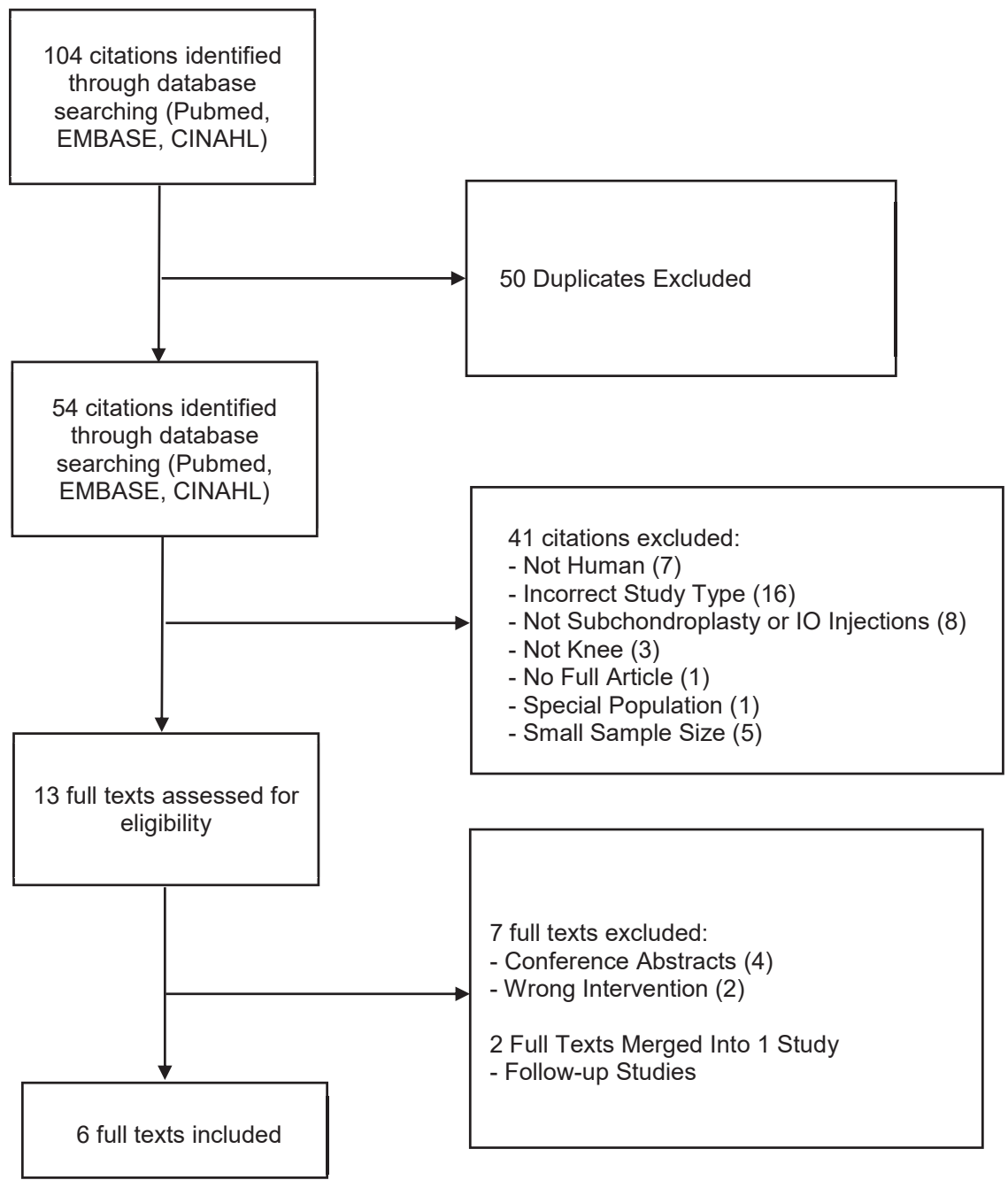

With regard to the study design, 2 (33 \%) studies provided Level II evidence, 2 (33\%) studies provided Level III evidence, and a further $2(33 \%)$ provided Level IV evidence. Five out of 6 studies reported data using the visual analog scale (VAS), 4 studies used the Knee Injury and Osteoarthritis Outcome Score (KOOS), while 3 studies used the Western Ontario and McMaster Universities Osteoarthritis (WOMAC) Index. Characteristics of the studies are summarized in Table 2.

\section{IO Injections of PRP}

Su et al. ${ }^{23}$ performed a three-way RCT comparing 1) IO injections of $2 \mathrm{~mL}$ of PRP, 2 administrations 14 days apart, 2) IA injections of $2 \mathrm{~mL}$ of PRP (2 administrations 14 days apart), and 3) IA injections of HA (5 administrations each 1 week apart). Mean platelet concentration in PRP $(789.68 \pm 17.80) \times 10^{9} / \mathrm{L}$ was 5.61-fold greater than that of whole blood $(140.73 \pm 11.26) \times 10^{9} / \mathrm{L}$. Mean leukocyte count in PRP $(29.92 \pm 1.54) 10^{\circ} / \mathrm{L}$ was 5.70 -fold greater than that of whole blood $(5.25 \pm 0.49) \times 10^{9} / \mathrm{L}$.
Sánchez et al. ${ }^{22}$ performed a comparative study between 2 different treatment modalities: 3 IA injections versus 1 subchondral injection of PRP followed by 2 IA injections of the same PRP preparation 1 week apart. With regard to subchondral injections, $5 \mathrm{~mL}$ of PRP was applied both at the femoral and tibial bone-cartilage interface, while $8 \mathrm{~mL}$ of PRP was used for IA delivery. PRP aliquots contained 2 to 3 times the concentration of platelets versus peripheral blood, depending on the hematocrit, platelet count and size, and was absent of erythrocytes and leukocytes.

Sánchez et al. $^{21}$ performed an observational study with 2 patient groups: 3 IA infiltrations of PRP on a weekly basis (IA group), versus a combination of 2 intraosseous PRP infiltrations with the first IA injection followed by 2 more IA injections in the following 2 weeks after the intraosseous infiltrations (IO Group). PRP preparations contained a moderate concentration of platelets ( 1.5 to 2.5 times the concentration of platelets compared with peripheral blood, depending on the platelet count and size, as well as the hematocrit) 


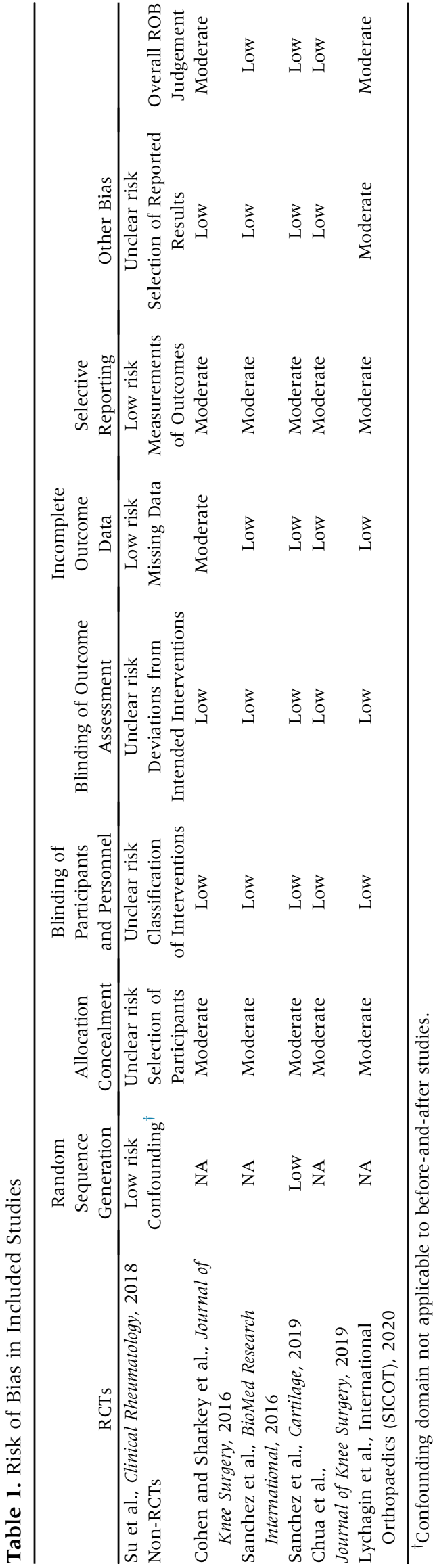

and was absent of erythrocytes and leukocytes. The PRP received by patients in the IA group contained a mean of $377.65 \pm 74.60$ platelets $/ \mathrm{mL}$ (range: $250-552$ platelets $/ \mathrm{mL}$ ), while that of the IO group contained a mean of $363.30 \pm 71.13$ platelets/mL (range: 198-518 platelets/ $\mathrm{mL}$ ).

Lychagin et al. ${ }^{19,20}$ performed a prospective cohort study where IO injections of $5 \mathrm{ml}$ of PRP product was compared against a control group of 17 otherwise healthy K-L grade I knee osteoarthritis (OA) patients without any clinical signs of OA or bone marrow lesions (BML). The composition and quality of the PRP were not reported.

\section{Subchondroplasty CaP Injections}

Chua et al. ${ }^{17}$ performed a prospective cohort study of 12 patients who underwent subchondroplasty under intraoperative radiographic guidance, for treatment of symptomatic BML in knee osteoarthritis. Postinjection, arthroscopy was performed to ensure that there was no extravasation of bone void filler into the knee joint, and to document IA injuries. No resurfacing, microfracture, or other cartilage regenerative procedures were performed.

Cohen and Sharkey ${ }^{18}$ performed a prospective cohort study of 66 patients who underwent subchondroplasty combined with arthroscopy, performed at a single center by one surgeon. Intraoperative fluoroscopic guidance was used. Arthroscopy was performed to aid in the accurate placement of the bone substitute, ensure that no IA extravasation of the injected material occurred, evaluate IA pathology, and address correctable problems. Patients with gross knee instability, or whose primary cause of pain and loss of function was due to pathology other than a BML, were excluded from the study to limit confounding factors.

\section{Clinical Outcomes}

\section{Visual Analog Score}

Details of the reported visual analog scale (VAS) scores are summarized in Table 3. The VAS scores reported by Lychagin et al. ${ }^{19,20}$ were reformatted from a 100-mm scale into a 10-point scale for easier comparison of results with other studies. With regard to the interpretation of the minimally clinically important difference (MCID) in pain, an improvement of 2 points or $20 \mathrm{~mm}$ was deemed as clinically important. ${ }^{24} \mathrm{In}$ addition, the baseline VAS scores are quite different among all 5 studies; therefore, the mean changes in VAS relative to the baseline was calculated. The mean decrease in VAS ranged from 3.31 to $4.91\left(4.3^{18}\right.$, $3.89^{22}, 4.91^{23}, 4.8^{17}$, and $3.31,{ }^{19,20}$ respectively) at 6 months postinjection and ranged from 4.03 to 5.4 $\left(4.73^{23}, 5.4^{17}\right.$, and $4.03,{ }^{19,20}$ respectively) at 12 months postinjection. In addition, $\mathrm{Su}$ et al. $^{23}$ describes the highest improvement in VAS at the 6-month time point; however, the VAS score was also noted to 
Table 2. Summary of Included Studies

\begin{tabular}{|c|c|c|c|c|c|c|c|}
\hline Study & $\begin{array}{l}\text { Level of } \\
\text { Evidence }\end{array}$ & Intervention & $\begin{array}{l}\text { Number of Patients in } \\
\text { Intervention Group }\end{array}$ & $\begin{array}{c}\text { Number of Patients } \\
\text { in Control Group }\end{array}$ & Follow-Up Period & $\begin{array}{l}\text { Pre/Post-operative } \\
\text { Imaging Modality }\end{array}$ & Complications \\
\hline $\begin{array}{l}\text { Cohen and Sharkey et al., } \\
\text { Journal of Knee Surgery, } 2016\end{array}$ & IV & Subchondroplasty & 66 & N.A. & Minimum 2 years & MRI & $\begin{array}{l}\text { 1/66 postoperative drainage at } \\
\text { CaP injection site } \\
\text { 1/66 postoperative DVT }\end{array}$ \\
\hline $\begin{array}{l}\text { Sánchez et al., BioMed Research } \\
\text { International, } 2016\end{array}$ & IV & PRP & $14(13$ assessed $)$ & N.A. & Minimum 18 Months & Not specified & $\begin{array}{l}\text { 1/14 Fever due to flu episode } \\
\text { 1/14 knee pain } 3 \text { months } \\
\text { after treatment } \\
\text { Both incidences likely } \\
\text { unrelated to treatment }\end{array}$ \\
\hline $\begin{array}{l}\text { Su et al., Clinical Rheumatology, } \\
2018\end{array}$ & II & PRP & $\begin{array}{c}28 \text { (IO and IA Injections) } \\
\text { (27 assessed) }\end{array}$ & $\begin{array}{l}\text { Two groups: } \\
26 \text { (IA Injections) } \\
(25 \text { assessed) } \\
32 \text { (HA injections) } \\
\text { (30 assessed) }\end{array}$ & Minimum 12 Months & Not specified & $\begin{array}{l}\text { Intervention: 1/28 Knee pain } \\
\text { and swelling } \\
\text { Control (IA): 1/26 knee } \\
\text { swelling, } 1 / 26 \text { knee pain } \\
\text { and swelling } \\
\text { Control (HA): } 1 / 32 \text { knee } \\
\text { pain, } 1 / 32 \text { knee swelling }\end{array}$ \\
\hline Sánchez et al., Cartilage, 2019 & III & PRP & 30 & 30 & Minimum 12 Months & Not specified & None \\
\hline $\begin{array}{l}\text { Chua et al., Journal of Knee } \\
\text { Surgery, } 2019\end{array}$ & II & Subchondroplasty & 12 & N.A. & Minimum 12 Months & MRI & $\begin{array}{l}1 / 12 \text { breakage of the cannula } \\
\text { within the bone during } \\
\text { removal }\end{array}$ \\
\hline $\begin{array}{l}\text { Lychagin et al., International } \\
\text { Orthopaedics (SICOT), } 2020\end{array}$ & III & PRP & 17 & 17 & Minimum 12 Months & MRI & None \\
\hline
\end{tabular}

CaP, calcium phosphate; DVT, deep vein thrombosis; HA, hyaluronic acid; IA: intraarticular; IO, intraosseous. 


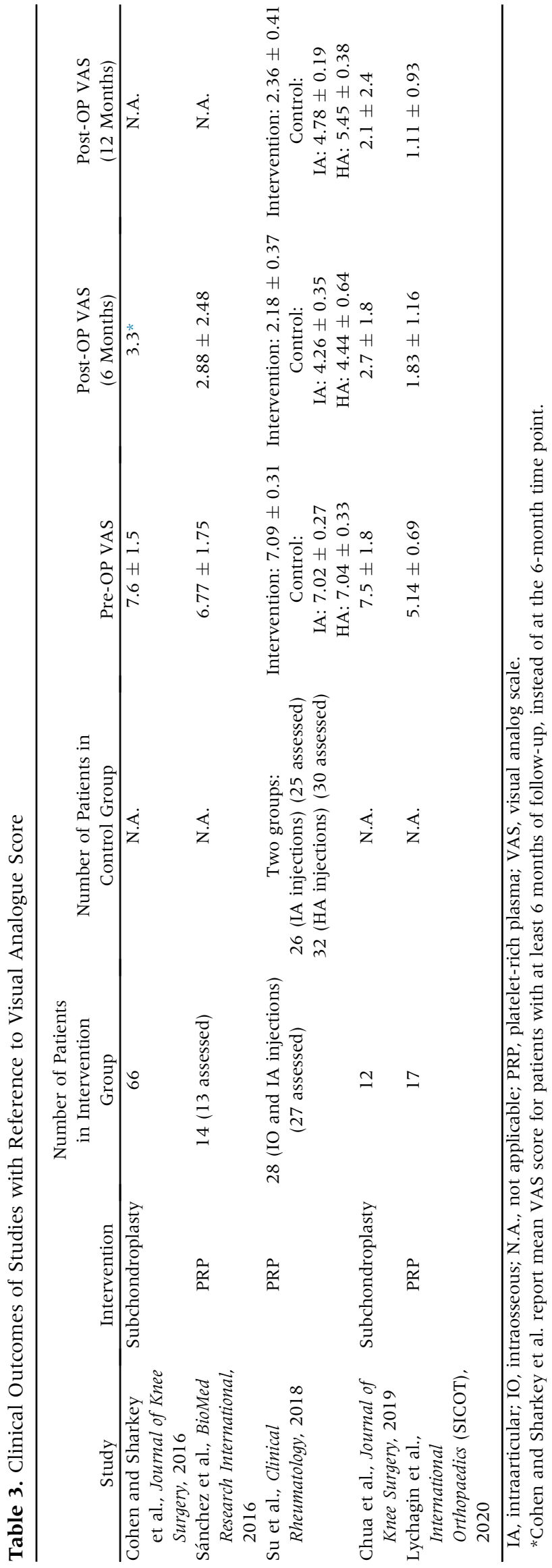

increase slightly by 0.18 points between the 6- and 12-month marks. In the other studies, VAS scores followed a consistently decreasing trend from baseline to 12 months postinjection.

\section{KOOS}

Details of the reported KOOS are summarized in Table 4. For KOOS, an increase of 8 to 10 points is considered clinically significant as the MCID. ${ }^{25}$ The mean changes in KOOS relative to the baseline were calculated. The mean increase in KOOS was $13.05^{22}$, $12.4^{21}$, and $22.34^{19,20}$ at 6 months postinjection, and $11.0^{21}, 34.7^{17}$, and $24.25^{19,20}$ at 12 months postinjection.

There were statistically significant improvements in the individual subcategories of the KOOS as well. These include activities of daily living, sports and recreation, and quality of life (QoL) scores. However, despite statistically significant improvements at 1 month following surgery $(P<.05)$, Lychagin et al. ${ }^{19,20}$ reported a statistically significant decrease $(P<.05)$ in average scores in all KOOS subsections apart from QoL, at 3, 6, and 12 months compared to 1 month following surgery. In the QoL subsection, a statistically significant increase $(P<.05)$ in average scores was observed at 3 months compared to 1 month following surgery.

\section{WOMAC}

Details of the reported WOMAC scores are summarized in Table 5. For WOMAC, an increase of 7.9 points is considered clinically significant as the MCID. ${ }^{24}$ Mean changes in WOMAC scores were calculated relative to the baseline. The decrease in WOMAC was $21.41^{23}$ and $10.87^{19,20}$ at 6 months postinjection, and $16.45^{23}$, $33.5,{ }^{17}$ and $9.72^{19,20}$ at 12 months postinjection.

\section{Complications}

A total of 6 patients out of 167 experienced adverse events related to the treatment modality. Cohen and Sharkey ${ }^{18}$ reported 1 patient (not reflected in Table 2 complications), who experienced postoperative drainage at the CaP injection site, resolved with surgical irrigation and debridement, and 1 patient diagnosed postoperatively with a deep vein thrombosis (not reflected in table as well), which required treatment with oral anticoagulation. Sánchez et al. ${ }^{22}$ reported 1 case of fever associated with flu, and 1 patient with an exacerbation of knee pain three months after treatment. Chua et al. ${ }^{17}$ reported 1 incidence of a breakage of the injective cannula within the bone, due to excessive knee manipulation. Su et al. ${ }^{23}$ reported 1 patient in the intervention group who presented with knee pain and swelling, and who subsequently withdrew from the study.

\section{Discussion}

The key finding reported in this study is an improvement in clinical outcomes post IO injection in 


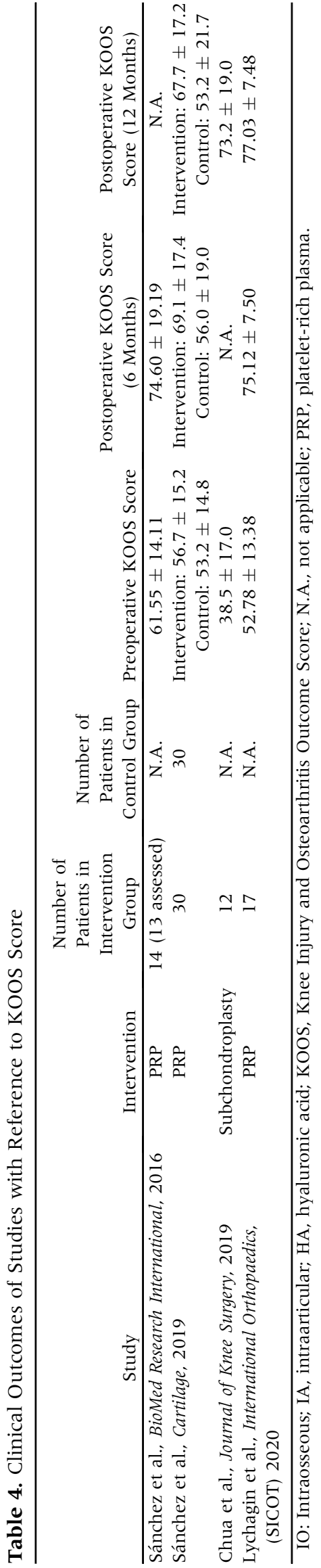

patients with knee OA based on VAS, KOOS, and WOMAC assessment tools. However, because of the lack of high-level evidence, follow-up, and heterogeneity of OA grades, it is difficult to ascertain the efficacy of IO injections of PRP and SCP, as standalone therapies in treating knee $\mathrm{OA}$ and in delaying progression to severe OA requiring TKR in the long term. Nonetheless, on the basis of our review, IO injections of PRP and SCP have demonstrated good safety profiles and improvement in clinical outcomes; therefore, it has potential to provide symptomatic relief and functional recovery in patients with knee OA. ${ }^{26}$

IO injections and SCP procedures were introduced on the basis that biologic agents, such as PRP, would be able to maintain tissue homeostasis and reduce inflammation while activating tissue healing pathways in the subchondral bone. ${ }^{27}$ On the other hand, SCP has also been noted to provide immediate mechanical support in the subchondral bone by the use of $\mathrm{CaP}$ ceramics, which help to reduce micromotion in the subchondral trabeculae, accounting for reported reduction in pain. ${ }^{28}$ In this review, outcomes of IO injection treatment modalities were assessed in relation to improvement in knee functionality and pain at baseline, 6 months, and 12 months postinjection. On the basis of the data reviewed, both modalities have been largely successful in improving patient outcomes, according to VAS, KOOS, and WOMAC scores. However, minor differences between IO injections of PRP and SCP might exist on the basis of the clinical outcomes measured. We noted that arthroscopy and targeted meniscus debridement in select cases were also performed in the same sitting as the $\mathrm{SCP}^{17}$ on the justification that arthroscopic debridement alone had not been proven to provide durable relief in arthritic knees. ${ }^{29}$ It is possible that this could result in differences in the actual VAS score, which would interfere with the reliability of the reported results. Ultimately, we feel that there is bound to be deviations and inconsistency in trending VAS scores due to the heterogeneity of patients. Moreover, activity levels of the patients after the operation were not tightly controlled and monitored, which could be a factor in triggering aggravation of the surgical site.

With regard to SCP procedures, it is interesting to note that a recent study performed by Chatterjee et al. ${ }^{30}$ demonstrated that outcomes based on KOOS and Tegner Lysholm Knee Scoring Scale scores improved significantly. However, despite the positive results, the procedure was surprisingly deemed "ineffective". This was based on the finding that nearly one-third of the study's patients showed "poor" clinical outcomes, according to the Tegner Lysholm scoring method. However, this method might be inappropriate for assessing the effectiveness of SCP injections, given that it has been used historically to evaluate the effectiveness of anterior cruciate ligament reconstructions. ${ }^{31,32}$ 


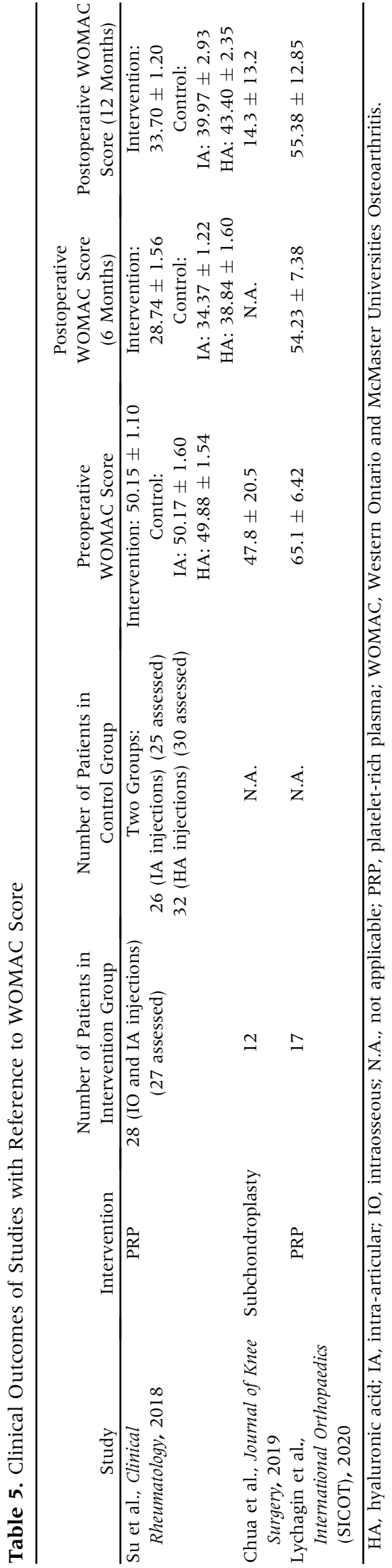

Concerning the procedural technique, we noted that Su et al. ${ }^{23}$ experienced difficulty in administering more than $2 \mathrm{~mL}$ of PRP solution intraosseously because of over-large resistance. This abnormality was not reported in the other studies being reviewed and could be due to suboptimal placement of the cannula within the $\mathrm{BML}$, as exact assessment of extension and location of the lesion is achieved by MRI; therefore, X-ray guidance can result in poor localization of the lesion, especially in relatively small BMLs. ${ }^{26}$ This raises the importance of correct localization of the subchondral lesion to avoid creating further complications in the joint and prolonging recovery time. Chua et al. ${ }^{17}$ suggested that clinical examination should be performed first to identify the point of maximum tenderness in the knee, followed by an MRI, to localize the site of subchondral lesion. Finally, a fluoroscope is used to guide the IO insertion of the trocar. The difficulty of IO injections is reinforced by the need for patient preparation, sedation, and training of the medical practitioner, making it more tedious and expensive than conventional IA injections, thus stressing the need for additional care when performing the procedure. ${ }^{33}$ Alternatively, novel strategies are being explored to implement software that can use preoperative MRI to calculate optimal placement of the cannula, which can be controlled by intraoperative computed tomography. ${ }^{26,34,35}$ However, more studies are required to evaluate the intraoperative outcomes and cost-benefit analysis of these technologies.

In addition, the presence of leukocytes in the PRP formulation is an area of controversy between the studies reviewed. Previous research suggested that leukocytes could have a possible detrimental effect by aggravating the proinflammatory environment in OA. ${ }^{36}$ It is suggested that leukocytes increase IL-1 activity and NF- $\kappa \beta$ expression, further promoting catabolic mechanisms and degradation of the osteochondral unit. However, Riboh et al. ${ }^{37}$ demonstrated that similar safety profiles exist for both leukocyte-rich PRP (LRPRP) and leukocyte-poor PRP (LP-PRP), and adverse reactions due to PRP might not be related to leukocyte concentrations. On the other hand, PRP preparations with LP-PRP have been reported to result in improved outcomes scores compared to LR-PRP, ${ }^{38}$ but the preparation of the LP-PRP might be on the grounds of efficiency rather than safety. ${ }^{21}$ Riboh et al. ${ }^{37}$ compared LP-PRP and LR-PRP in the treatment of knee OA, with LP-PRP injections, resulting in significantly improved WOMAC scores compared to HA or placebo. ${ }^{39-43}$ Filardo et al. studied LR-PRP injections and found no statistical difference with HA injections, providing further evidence that LP-PRP may be the preferred preparation for the treatment of osteoarthritis symptoms. ${ }^{39,44}$ Despite the various studies on LR-PRP and LP-PRP, there is currently no consensus on the optimal 
PRP preparation with respect to blood components because of the many commercial variants available in the market ${ }^{39}$ and the lack of clinical trials. Nonetheless, there is a need for a scientific and standardized approach in analyzing PRP formulation composition, which can be carried out by standardization of PRP preparation methodology and detailed characterization of the final PRP product. ${ }^{45}$ In addition, future studies specifically comparing the clinical outcomes of LR-PRP and LP-PRP could be done to better understand the differences between both preparations.

Assessment of any treatment modality involves examining the associated risks. Commonly reported risks associated with IO injections and SCP involve postinjection swelling, possibly because of infection or thrombosis, as well as leakage of biologics into the IA space. In terms of management, knee swelling and postoperative pain are common complications that can be controlled by multimodal analgesia to reduce inflammation. ${ }^{46}$ On the other hand, leakage of biologics into the IA space is more concerning, as it could create a prolonged state of inflammation in the joint space, which will slow healing and cause further damage to the joint. This incidence of this complication can be reduced by proper risk profiling in the case of osteoporotic patients, who are more susceptible, as well as proper administration of the cannula into the subchondral bone. $^{26}$ This review showcased that the complication rate for both procedures was low and did not lead to any long-term adverse outcomes. Preoperative imaging could be a critical factor in the success of these procedures, as correct identification of the target site would better facilitate the administration of the cannula and provide smoother access to the subchondral bone. That being said, the extraction of the cannula should be performed meticulously as well to avoid aggravation of surrounding tissue and formation of clots, which would complicate the healing process. Apart from the overall low risks of IO injections in SCP, cost effectiveness and practicality are important considerations. The process of obtaining PRP is effort-dependent, and requires careful extraction and refinement of the patient's blood. In addition, the equipment costs incurred in producing a sample of PRP might be relatively higher than SCP, which uses CaP, a readily available product in most clinical settings. ${ }^{26}$ As such, clinical effectiveness needs to be weighed against cost effectiveness to achieve the most practical and efficacious care for patients.

Finally, OA is a heterogeneous and multifactorial pathology and the underlying mechanisms causing the disease might be different in each patient. ${ }^{38}$ Phenotypes of OA include senescent, inflammatory, metabolic, genetic, and endocrine. PRP-derived products, which are anti-inflammatory, might be useful in treating the inflammatory phenotype, but might not be as useful in addressing other phenotypes. Therefore, it is important to stratify patients according to the pathogenicity of knee OA, so that appropriate and specific treatment options can be selected. With regard to imaging, MRI is among the key imaging techniques used to stratify and monitor patients with changes in bone, cartilage, and inflammation. Biochemical markers have also been used as secondary parameters, and thus, they could also be helpful to further delineate OA phenotypes. ${ }^{47}$

\section{Limitations}

The findings discussed in this review should be carefully considered alongside the limitations of the current literature. These include the small number of studies available, the small number of patients included in the studies (total 167), and the lack of postinjection imaging (including MRI) to evaluate the healing potential of the treatment. Furthermore, the lack of randomized controlled trials on the subject is apparent (only 1 included in the literature). It is also worth considering that clinically significant changes in pain are not uniform along the entire VAS, ${ }^{48}$ as the evaluation of pain is ultimately subjective to each patient. Another limitation is the need for research on the effect of IO injections on cartilage repair and regeneration. None of the included studies reported results in this area. These would give relevant insight into the correlations between subchondral bone pathology and the health of the articular cartilage. Besides, it is a challenge to gain an accurate assessment of the monetary costs of different treatment modalities across the studies originating from various research and medical centres. This would be important information in the final costbenefit assessment of each treatment modality. A possible extension of the subject could be an evaluation into the cost-effectiveness of the different IO injection treatment modalities. This would be pertinent, given the importance of quality, yet affordable, regimes in the treatment of knee pathologies, including osteoarthritis.

\section{Conclusion}

Intraosseous injections for knee osteoarthritis are safe and effective. However, multiple pertinent variables, such as safety, cost of treatment, and performance against placebos and other treatment modalities require further evaluation before intraosseous injections can be considered as standard treatment for patients presenting with osteoarthritis of the knee.

\section{References}

1. Litwic A, Edwards MH, Dennison EM, Cooper C. Epidemiology and burden of osteoarthritis. $\mathrm{Br}$ Med Bull 2013;105:185-199. doi:10.1093/bmb/lds038.

2. Xie F, Thumboo J, Fong KY, et al. A study on indirect and intangible costs for patients with knee osteoarthritis in Singapore. Value Health 2008;11:S84-S90. doi:10.1111/j. 1524-4733.2008.00371.x (Suppl 1). 
3. Vos T, Flaxman AD, Naghavi M, et al. Years lived with disability (YLDs) for 1160 sequelae of 289 diseases and injuries 1990-2010: A systematic analysis for the Global Burden of Disease Study 2010. The Lancet 2012/12/15/ 2012;380:2163-2196. https://doi.org/10.1016/S01406736(12)61729-2.

4. World Health Organization. WHO methods and data sources for global burden of disease estimates 2000-2011. Global Health Estimates Technical Paper. 2013;WHO/HIS/ HSI/GHE/2013.4.

5. Losina E, Weinstein AM, Reichmann WM, et al. Lifetime risk and age at diagnosis of symptomatic knee osteoarthritis in the US. Arthritis Care Res (Hoboken) 2013;65: 703-711. doi:10.1002/acr.21898.

6. Driban JB, Harkey MS, Liu S-H, Salzler M, McAlindon TE. Osteoarthritis and aging: Young adults with osteoarthritis. Curr Epidemiol Rep 2020;7:9-15. doi:10.1007/s40471-02000224-7.

7. World Health Organization. Obesity and overweight. World Health Organization. https://www.who.int/news-room/ fact-sheets/detail/obesity-and-overweight. [Accessed March 21, 2021].

8. Bayliss LE, Culliford D, Monk AP, et al. The effect of patient age at intervention on risk of implant revision after total replacement of the hip or knee: A population-based cohort study. Lancet 2017;389:1424-1430. doi:10.1016/ s0140-6736(17)30059-4.

9. Donell S. Subchondral bone remodelling in osteoarthritis. EFORT Open Rev 2019;4:221-229. doi:10.1302/2058-5241. 4.180102.

10. Kon E, Buda R, Filardo G, et al. Platelet-rich plasma: Intraarticular knee injections produced favorable results on degenerative cartilage lesions. Knee Surg Sports Traumatol Arthrosc 2010; 18:472-479. doi:10.1007/s00167-009-0940-8.

11. Chang KV, Hung CY, Aliwarga F, Wang TG, Han DS, Chen WS. Comparative effectiveness of platelet-rich plasma injections for treating knee joint cartilage degenerative pathology: A systematic review and meta-analysis. Arch Phys Med Rehabil 2014;95:562-575. doi:10.1016/j. apmr.2013.11.006.

12. Bottegoni C, Dei Giudici L, Salvemini S, Chiurazzi E, Bencivenga $\mathrm{R}$, Gigante A. Homologous platelet-rich plasma for the treatment of knee osteoarthritis in selected elderly patients: An open-label, uncontrolled, pilot study. Ther Adv Musculoskelet Dis 2016;8:35-41. doi: 10.1177/1759720x16631188.

13. Cole BJ, Seroyer ST, Filardo G, Bajaj S, Fortier LA. Platelet-rich plasma: Where are we now and where are we going? Sports Health 2010;2:203-210. doi:10.1177/ 1941738110366385.

14. Moher D, Liberati A, Tetzlaff J, Altman DG, PRISMA Group. Preferred reporting items for systematic reviews and meta-analyses: the PRISMA statement. PLoS Med 2009;6. doi:10.1371/journal.pmed.1000097. el000097e 1000097.

15. Higgins JP, Altman DG, Gøtzsche PC, et al. The Cochrane Collaboration's tool for assessing risk of bias in randomised trials. BMJ 2011;343:d5928. doi:10.1136/bmj. d5928.

16. Sterne JA, Hernán MA, Reeves BC, et al. ROBINS-I: A tool for assessing risk of bias in non-randomised studies of interventions. BMJ 2016;355:i4919. doi:10.1136/bmj. i4919.

17. Chua K, Kang JYB, Ng FDJ, et al. Subchondroplasty for bone marrow lesions in the arthritic knee results in pain relief and improvement in function. J Knee Surg 2021;34: 665-671. doi:10.1055/s-0039-1700568.

18. Cohen SB, Sharkey PF. Subchondroplasty for treating bone marrow lesions. J Knee Surg 2016;29:555-563. doi: 10.1055/s-0035-1568988.

19. Lychagin A, Lipina M, Garkavi A, et al. Intraosseous injections of platelet-rich plasma for knee bone marrow lesions treatment: One-year follow-up. Int Orthop 2021;45:355-363. doi:10.1007/s00264-020-04546-5.

20. Lychagin AV, Garkavi AV, Islaieh OI, et al. Effectiveness of intraosseous infiltration of autologous platelet-rich plasma in the area of the bone marrow edema in osteoarthritis of the knee joint. Bull Russ State Med Univ 2019;8: 47-53. doi:10.24075/brsmu.2019.053.

21. Sánchez M, Delgado D, Pompei O, et al. Treating severe knee osteoarthritis with combination of intra-osseous and intra-articular infiltrations of platelet-rich plasma: An observational study. Cartilage 2019;10:245-253. doi:10. $1177 / 1947603518756462$.

22. Sánchez M, Delgado D, Sánchez P, et al. Combination of intra-articular and intraosseous injections of platelet-rich plasma for severe knee osteoarthritis: A pilot study. Biomed Res Int 2016;2016:4868613. doi:10.1155/2016/4868613.

23. Su K, Bai Y, Wang J, Zhang H, Liu H, Ma S. Comparison of hyaluronic acid and PRP intra-articular injection with combined intra-articular and intraosseous PRP injections to treat patients with knee osteoarthritis. Clin Rheumatol 2018;37:1341-1350. doi:10.1007/s10067-018-3985-6.

24. Tubach F, Ravaud P, Baron G, et al. Evaluation of clinically relevant changes in patient reported outcomes in knee and hip osteoarthritis: The minimal clinically important improvement. Ann Rheum Dis 2005;64:29-33. doi:10.1136/ard.2004.022905.

25. Roos EM, Lohmander LS. The Knee injury and Osteoarthritis Outcome Score (KOOS): From joint injury to osteoarthritis. Health Qual Life Outcomes 2003;1. doi:10. 1186/1477-7525-1-64.

26. Di Matteo B, Polignano A, Onorato F, et al. Knee intraosseous injections: A systematic review of clinical evidence of different treatment alternatives. Cartilage 2020. doi:10.1 177/1947603520959403:1947603520959403.

27. Ramaswamy Reddy SH, Reddy R, Babu NC, Ashok GN. Stem-cell therapy and platelet-rich plasma in regenerative medicines: A review on pros and cons of the technologies. J Oral Maxillofac Pathol 2018;22:367-374. doi:10.4103/ jomfp.JOMFP_93_18.

28. Farr J, Cohen SB. Expanding applications of the subchondroplasty procedure for the treatment of bone marrow lesions observed on magnetic resonance imaging. Operative Techniques Sports Med 2013;21:138-143. doi:10. 1053/j.otsm.2013.03.006.

29. Thorlund JB, Juhl CB, Roos EM, Lohmander LS. Arthroscopic surgery for degenerative knee: Systematic review and meta-analysis of benefits and harms. BMJ 2015;350:h2747. doi:10.1136/bmj.h2747.

30. Chatterjee D, McGee A, Strauss E, Youm T, Jazrawi L. Subchondral calcium phosphate is ineffective for bone 
marrow edema lesions in adults with advanced osteoarthritis. Clin Orthop Relat Res 2015;473:2334-2342. doi:10. 1007/s1 1999-015-4311-0.

31. Mitsou A, Vallianatos P, Piskopakis N, Maheras S. Anterior cruciate ligament reconstruction by over-the-top repair combined with popliteus tendon plasty. J Bone Joint Surg Br May 1990;72:398-404. doi:10.1302/0301620x.72b3.2341436.

32. Bengtsson J, Möllborg J, Werner S. A study for testing the sensitivity and reliability of the Lysholm knee scoring scale. Knee Surg Sports Traumatol Arthrosc 1996;4:27-31. doi:10.1007/bf01565994.

33. Sánchez M, Fiz N, Guadilla J, et al. Intraosseous infiltration of platelet-rich plasma for severe knee osteoarthritis. Arthrosc Tech 2014;3:e713-e717. doi:10.1016/j.eats.2014. 09.006.

34. Hoffmann R, Thomas C, Rempp H, et al. Performing MRguided biopsies in clinical routine: Factors that influence accuracy and procedure time. Eur Radiol 2012;22: 663-671. doi:10.1007/s00330-011-2297-x.

35. Weiss CR, Nour SG, Lewin JS. MR-guided biopsy: A review of current techniques and applications. J Magn Reson Imag 2008;27:31 1-325. doi:10.1002/jmri.21270.

36. Assirelli E, Filardo G, Mariani E, et al. Effect of two different preparations of platelet-rich plasma on synoviocytes. Knee Surg Sports Traumatol Arthrosc 2015;23: 2690-2703. doi:10.1007/s00167-014-3113-3.

37. Riboh JC, Saltzman BM, Yanke AB, Fortier L, Cole BJ. Effect of leukocyte concentration on the efficacy of platelet-rich plasma in the treatment of knee osteoarthritis. Am J Sports Med 2016;44:792-800. doi:10.1177/ 0363546515580787.

38. Gato-Calvo L, Magalhaes J, Ruiz-Romero C, Blanco FJ, Burguera EF. Platelet-rich plasma in osteoarthritis treatment: review of current evidence. Ther Adv Chronic Dis 2019; 10: 2040622319825567. doi:10.1177/2040622319825567.

39. Le ADK, Enweze L, DeBaun MR, Dragoo JL. Current clinical recommendations for use of platelet-rich plasma.
Curr Rev Musculoskelet Med 2018;11:624-634. doi:10.1007/ s12178-018-9527-7.

40. Cerza F, Carnì S, Carcangiu A, et al. Comparison between hyaluronic acid and platelet-rich plasma, intra-articular infiltration in the treatment of gonarthrosis. Am J Sports Med 2012;40:2822-2827. doi:10.1177/0363546512461902.

41. Sánchez M, Fiz N, Azofra J, et al. A randomized clinical trial evaluating plasma rich in growth factors (PRGFEndoret) versus hyaluronic acid in the short-term treatment of symptomatic knee osteoarthritis. Arthroscopy 2012;28:1070-1078. doi:10.1016/j.arthro.2012.05.011.

42. Patel S, Dhillon MS, Aggarwal S, Marwaha N, Jain A. Treatment with platelet-rich plasma is more effective than placebo for knee osteoarthritis: A prospective, doubleblind, randomized trial. Am J Sports Med 2013;41: 356-364. doi:10.1177/0363546512471299.

43. Hart R, Safi A, Komzák M, Jajtner P, Puskeiler M, Hartová P. Platelet-rich plasma in patients with tibiofemoral cartilage degeneration. Arch Orthop Trauma Surg 2013;133:1295-1301. doi:10.1007/s00402-013-1782-x.

44. Filardo G, Di Matteo B, Di Martino A, et al. Platelet-rich plasma intra-articular knee injections show no superiority versus viscosupplementation: A randomized controlled trial. Am J Sports Med 2015;43:1575-1582. doi:10.1177/ 0363546515582027.

45. Piuzzi NS, Chughtai M, Khlopas A, et al. Platelet-rich plasma for the treatment of knee osteoarthritis: A review. J Knee Surg 2017;30:627-633. doi:10.1055/s-0037-1603795.

46. Li J-W, Ma Y-S, Xiao L-K. Postoperative pain management in total knee arthroplasty. Orthop Surg 2019;11: 755-761. doi:10.1111/os.12535.

47. Van Spil WE, Kubassova O, Boesen M, Bay-Jensen A-C, Mobasheri A. Osteoarthritis phenotypes and novel therapeutic targets. Biochem Pharmacol 2019;165:41-48. doi: 10.1016/j.bcp.2019.02.037.

48. Bird SB, Dickson EW. Clinically significant changes in pain along the visual analog scale. Ann Emerg Med 2001;38:639-643. doi:10.1067/mem.2001.118012. 